\title{
Equine Tendinopathy Therapy Using Mesenchymal Stem Cells
}

\section{Svetlana Platonova, Daria Korovina, Ekaterina Viktorova, and Irina Savchenkova}

Federal State Budget Scientific Institution "Federal Scientific Center VIEV”, Moscow, Russia

ORCID:

Svetlana Platonova: http://orcid.org/0000-0001-5946-9071

\section{Abstract}

The application of animal mesenchymal stem cells has been actively studied in veterinary medicine over the past decade. In horses, the use of stem cells is mainly aimed at the treatment and maintenance of the musculoskeletal system. This review summarizes the currently published data on the therapeutic use of mesenchymal stem cells in equine tendon injuries. Tendon lesions are the most common cause of horse limp. It has been demonstrated that the use of mesenchymal stem cells compared with traditional methods of treatment can significantly reduce the re-injury. Currently, there

Corresponding Author: Svetlana Platonova s.vasileva89@yandex.ru

Published: 5 April 2021

Publishing services provided by Knowledge E

(c) Svetlana Platonova et al. This article is distributed under the terms of the Attribution License, which permits unrestricted use and redistribution provided that the original author and source are credited.

Selection and Peer-review unde the responsibility of the DonAgro Conference Committee.

\section{G OPEN ACCESS} are a large number of stem cell application protocol versions. Many researchers have obtained positive clinical results in using a combination of mesenchymal stem cells and platelet-rich blood plasma. Studies have demonstrated the safety of using allogeneic mesenchymal stem cells as an alternative to autologous. This review shows that the application of mesenchymal stem cells for horse tendon regeneration is a perspective area in veterinary medicine.

\section{Introduction}

Among all injuries of equine musculoskeletal system, tendon injuries take up $46 \%$ and are more specific for athletic equines $[1,2]$. The healing process of the tendon is slow and often characterized by the formation of a fibrous scar different from the tendon by its structure and having worse mechanical properties which in $67 \%$ lead to re-injury [3]. In this regard, the optimal treatment of tendon injuries should be aimed at the regeneration of their structure and functions. Currently, there are a number of surgical and therapeutic approaches for treating both acute and chronic tendon injuries. However, they are very often ineffective and lead to long periods of rehabilitation and relapse. In recent years, mesenchymal stem cells (MSCs) are of great interest as a one treatment option. MSCs are a self-renewing population of multipotent cells that can differentiate into mesoderm cells and regenerate the damaged tissues, which makes them vital for any living system 
[4]. Due to their availability, ability to reduce inflammation and accelerate healing, MSCs have essential therapeutic applications. Equine MSCs were isolated from bone marrow (BM) [5-7], adipose tissue (AT) [8-10], peripheral blood [11, 12] synovial membrane and synovial fluid [13], umbilical cord blood (UCB) [10,14-18]. The selection of MSCs source depends on the easiness of access and collection of material, the need for local or general anesthesia, and the final concentration and quality of isolated cells. The most significant sources of MSCs in clinical practice are BM, AT, and UCB [19]. Currently, there are a large number of publications describing the clinical advantages of MSCs compared to traditional therapy in equine. It has been shown that the use of MSCs for regenerative purposes has great potential. Therefore it is very important to analyze their advantages and disadvantages before using [20]. This review summarizes the published data on the therapeutic use of equine MSCs while tendon injuries.

\section{Methods and Equipment}

The damage of the superficial digital flexor tendon, SDFT is the most common cause of lameness in horses [21]. In 2003, MSCs were first injected into damaged SDFT horses. The BM-MSCs have been used as they are most studied and characterized. Smith et al. have injected into the injured tendon of a horse a $6.4 \times 105$ autologous BM-MSCs. As a result of the study, in horse oedema of limb or lameness was not noticed, only pain with pressure was observed. Ultrasound also did not reveal pathologies in the healing of the tendon [5]. Later, Godwin et al. analyzed the use of autologous BMMSCs for the treatment of SDFT injuries in 141 horses. The MSCs have been obtained from sternum BM cultivated for 14 days and cryopreserved. A cell suspension with a $5 \times 106$ cells/ml concentration of BM-MSCs was injected by the injection needle into the damaged area under the ultrasound control. The number of injection sites was not standardized and depended on the extent and size of the injury visualized by ultrasound. In most cases, it was 2-4 injections over the entire area of the lesion. According to the results of a three-year observation, out of 113 horses, 111 (98.2 \%) returned to the competition; 31 horses (27.4\%) suffered repeated injuries, and only 6 horses (5.3\%) were injured in the contralateral limb. The average number of injected cells was $9.2 \times 106$ for horses that did not suffer and $7.6 \times 106$ cells for those who suffered from re-injury. But there were no statistically significant differences. When using BMMSCs, the frequency of re-injuries was significantly lower $(27.4 \%)$ than when treated with hyaluronan or polysulfated glycosaminoglycans, where $56 \%$ of horses have reinjury within 2 years $[3,7]$. The similar results were previously obtained by Smith et al. 
when using autologous BM-MSCs in the treatment of 168 horses with the medium and high degree severity of SDFT injuries. At that time the frequency or re-injury was $18 \%$ [6]. More lately, Smith et al. have demonstrated that injection of autologous BM-MSCs suspended in the BM supernatant results in the formation of the tissue is more similar to a normal tendon than scar tissue. For this study, 12 horses with natural SDFT injury were randomly divided into experimental and control groups. The control group received an intracranial injection of $2 \mathrm{ml}$ saline and the experimental group was injected of 10×106 BM-MSCs suspended in $2 \mathrm{ml}$ of BM supernatant. The side effects were not noted. After 6 months, the horses were euthanized, and the tendons were evaluated. As a result, it was shown that in contrast to physiological saline, the injection of BM-MSCs with BM supernatant facilitate normalization of following parameters as stiffness, hydration, contents of glycosaminoglycan and histological organization [22]. It has to be mentioned that in this study it is impossible to determine what caused the effect: BM-MSCs, BM supernatant or their complex.

It is known that the AT by their availability is an appealing source of MSCs, and therefore Nixon et al. in 2008 published the first article on the effect of fractions of nucleated AT cells on the regeneration of induced SDFT damages of horses [8]. The study was short-term and 6 weeks after the injection, the horses were euthanized. Histological evaluation showed a restoration in the structure of the tendons of the experimental group. However, the differences in biochemical and molecular parameters compared with the control were insignificant [8]. Later, Carvalho et al. conducted an examination of purified and characterized autologous AT-MSCs on the horses induced SDFT injuries. Eight animals were at once injected with $10 \times 106$ AT-MSCs into the tendons, with no side effects. Histological analysis showed that AT-MSCs are equable to regenerate the tendon, reduce inflammatory infiltrate, and increase expression of type I collagen [9]. Ahrberg et al. conducted a blind randomized study to analyze the influence of autologous AT-MSCs in SDFT tendinopathies induced on all limbs of 6 horses [23]. Tendinopathies were caused by an insignificant mechanical rupture of tissues in combination with an injection of a low dose of type I collagenase (4.8 $\mathrm{mg} / \mathrm{ml}$ ) into the tendons of all extremities. MSCs were isolated from AT cultured up to 2 passages and were labelled with particles of supermagnetic iron oxide. Three weeks after injury, labelled AT-MSCs were injected into the lesions under ultrasound guidance. In a randomized way, autologous serum contained AT-MSCs in a concentration of $10 \times 106$ cells $/ \mathrm{ml}$ was injected into one hind limb and one forelimb. In a contralateral side was injected an autologous blood serum without MSCs. Tendons that were injected with AT-MSCs demonstrated the long-term clinical signs of inflammation, including swelling, 
fever, and pain. Twenty four weeks after the injection of MSCs animals were euthanized. All the tendons were then prepared, analyzed and sent for histological examination. The authors did not find significant differences in histological, biochemical or biomechanical parameters between lesions treated with AT-MSCs and control lesions of tendons treated with blood serum. However, the hydroxylysylpyridinoline composed in the MSCs group is more inherent to the healthy tendon tissue which potentially indicates better healing. It has also been demonstrated that labelled MSCs integrate from the injection site into the lesion, which is in accordance with previously published studies [23, 24]. However, there have been published studies that did not determine the obvious impact of AT-MSCs. For instance, Geburek et al. were conducted a randomized controlled blind experimental study. For this purpose, 9 horses of 3-6 years old that did not manifest the signs of SDFT disturbances before the experiment beginnings were selected. Near the lesion site, each horse was injected ( 3 and $5 \mathrm{~cm}$ proximal to the surgical scar on the skin) by $0.5 \mathrm{ml}$ of inactivated autologous serum contained AT-MSCs at a concentration of $10 \times 106$ cells $/ \mathrm{ml}$, or only inactivated autologous serum that served as a control. The injection was made under ultrasound guidance. The injection of AT-MSCs did not significantly influence on clinical and ultrasound parameters. At 24 weeks after the start of treatment, the biochemical, histological, and biomechanical characteristics of the reparative tissue did not have essential differences between the groups [25].

An alternative source of equine MSCs is UCB, which can be used for obtaining MSCs in a minimally invasive way. In 2013, Kang et al. analyzed allogeneic UCB-MSCs therapy while natural SDFT injury of horses. Totally, six animals aged from 3 to 5 years were involved in the study. Each animal was injected with $2 \times 107$ cells suspended in $10 \mathrm{ml}$ of phosphate-buffered saline (PBS). Adverse reactions from the introduction of cells were not noted. An ultrasound was performed after 1, 2, 4, 8, and 12 weeks. One month after the UCB-MSCs injection while ultrasound detected a decrease in the lesion site of SDFT and 3 months after the tendons were almost completely regenerated [15]. Van Loon et al revealed a positive effect of UCB-MSCs on the healing of horse tendon injuries in their studies. Scientists analyzed the effects of UCB-MSCs cell therapy on SDFT tendonitis and deep digital flexor tendon (DDFT) tendons [16]. Allogeneic MSCs isolated from UCB by centrifugation at ficoll density were used for injection. One dose consisted of $3 \mathrm{ml}$ of supernatant and included approximately $10 \times 106$ cells. For one lesion, about $(2-10) \times 106$ cells were used. The number of injection areas was not standardized and depended on the size and structure of the lesion. In local lesions, injections were introduced from one place, in diffusion lesions by the $5 \mathrm{~mm}$ intervals. Due to the severity of the pathology and the lack of the expected result, after the first injection, to one horse out of 29 
UCB-MSCs were double injected. Twenty-four of 29 horses (83\%), six months after the injection, were able to return to training at the same or higher level [16].

Along with the MSCs application studies as cell therapy, new publications on the possibility of equine tendon injuries, treatment by the platelet-rich plasma (PRP) and platelet concentrate (platelet concentrate, PC) are appearing.

These studies demonstrate that PRP and PC are able to provide important biological effects as increased production of collagen type I, the proliferation of tenocytes, hagiogenesis, and the organization and fibres alignment [26, 27]. Del Bue M. was one of the first to analyze the use of a complex of allogeneic AT-MSCs and PRP in horse tendon injury. The animals participating in the study were heterogeneous in age, activity, and gender. As a result, out of 16 horses with tendonitis 14 returned to work (87.5\%) and remained active during the 2 year observation period, two horses did not recover, one of them suffered from chronic tendonitis and the other returned to training too early and consequently, the injury relapsed. During the postoperative recovery, $50 \%$ of horses had no complications, except the mild local edema and increased pain on the second day which is probably associated with tissue inflammation from intracranial injection [26]. Later Carvalho Ade et al. showed that injection of AT-MSCs in conjunction with PC while inducing SDFT injury in horses leads to the regeneration of collagen fibers and a reduction in the inflammatory infiltrate. In the investigation, eight animals have been divided into two equal groups and studied. The experimental group received intracranial injections of $10 \times 106$ AT-MSCs suspended in $1 \mathrm{ml}$ of autologous PC with an average concentration of $321 \times 103$ platelets / $\mu \mathrm{L}$, the control group received a similar amount of PBS. Side effects were not observed. An ultrasound examination of the tendons was carried out every two weeks and showed the absence of progression of the lesion. After 16 weeks, histological, immunohistochemical parameters and gene expression were evaluated using SDFT biopsy [28]. The results are consistent with the studies that used MSCs without platelet concentrate on equine tendonitis treatment [9, 25]. Ricco et al. confirmed the safety and efficacy of therapy based on the use of allogeneic ATMSCs in conjunction with PRP. This complex was tested on 19 horses suffering from acute or subacute SDFT tension. The allogeneic AT-MSCs application did not cause clinical signs of acute or chronic side effects, and also the formation of pathological tissue during the long-term examination was absent. After 24 months of observation, $89.5 \%$ of the horses returned to their original training level and the frequency of reinjury was $10.5 \%$, which is comparable to the data obtained for autologous MSCs [29]. Later Guercio et al. have also tested cell therapy for AT-MSCs in combination with PRP for the equine SDFT injuries. Nine sports horses aged 3 to 12 years were selected with 
SDFT lesions on the forelimb. All horses under ultrasound control were injected with AT-MSCs in conjunction with PRP. The number of injected MSCs was $1 \times 106$ cells in 5-10 $\mathrm{ml}$ (depending on the severity and extent of the lesion) with a platelet concentration of $1 \times 109 \mathrm{cells} / \mathrm{ml}$. After injection, the animals immediately showed slight edema which passed after 48 hours. Thirteen days after the injection, an ultrasound scan noticed an increase of echogenicity, intralesionality, invariability of the lesion size. After 60 days, the size of the lesion decreased. Full alignment of tendon fibers was revealed after 120 days. After 7-9 months, 7 of 9 horses (78\%) were able to return to their previous activity without relapse. Two horses out of 9 (22 \%) have got repeatedly injured. However, it has to be mentioned that in one horse a relapse occurred in the same tendon but in a different place; in the second horse, relapse was the result of a violation of the rehabilitation protocol. It is believed that postoperative rehabilitation of the patient is a necessary support for the proper tendon remodelling. In this study, it was noted that a rehabilitation time less than 6 months increases the relapse rate [30]. Later Romero et al. compared the use of autologous BM-MSCs, AT-MSCs and PRPs for equine SDFT injuries treatment. Injuries were surgically created on both forelimbs of 12 horses, thus four groups of six damaged tendons in each were formulated. One of the groups served as a control, the tendons of this group were processed by Ringer's solution with lactate. The ultrasound examination was performed before injection and 2, 6, 10, 20 and 45 weeks after. After 45 weeks, histological analysis and gene expression analysis were performed. The results showed that compared with the control group, all types of treatment have a clear positive effect. Injection of BM-MSCs led to a better effect than PRP or AT-MSCs, however, the differences between treatment results were relatively small [31].

Application of predifferentiated MSCs is one of the ways of reducing the risk of objectionable differentiation of injected into the inflamed tendon cells. A team led by $\mathrm{C}$. Beerts analyzed the effect of introducing allogeneic, tenogenous, pre-differentiated MSCs in combination with PRP on 36 SDFT damaged horses [11]. MSCs were obtained from the peripheral blood of a 6 year old German Warmblood horse and pre-differentiated by adding tenogenic growth factors to the culture medium until the markers of tenogenic differentiation were increased. The number of injected MSCs and PRP per injury was composed $(2-3) \times 106$ cells and (1-1.5) $\times 108$ platelets, respectively. The same dose was used for all sizes of lesions. After the injection, no adverse reactions were noted. However, non-steroidal anti-inflammatory drugs were administered to the horses within 3-5 days after the injection which could reduce possible edema. Horses re-injected with MSCs were $33.3 \%$. Twenty-four months after the onset of treatment, $85.7 \%$ of the 
horses were able to return to the competition to their original level. The relapse rate after 12 months was $12.5 \%$ and after 24 months $14.3 \%$, which is comparable with the data obtained using undifferentiated MSCs in combination with PRP [11, 28, 29]. The results of this study are promising, however, it should be noted that there is no control group in the study, and a group receiving only MSCs, which makes it impossible to estimate the therapeutic potential of MSCs compared to that of undifferentiated MSCs.

\section{Conclusion}

The tendon injuries in horses are widespread and due to the long-lasting treatment, a high possibility of relapsing, cause problems for horse breeding. Currently, a large number of studies dedicated to the injection of MSCs as an alternative to traditional treatments demonstrating the efficacy of MSCs in equine tendon injuries treatment have been collected. The review of the literature shows that despite a wide variety of methods, in general, the results of treating horse tendinopathies with MSCs are consistent and show better healing of tendons, as well as a decrease in the frequency of re-injuries compared to traditional treatments. Thus, according to the results of a twoyear study conducted by Dyson, the relapse rate in the traditional treatment of equine SDFT inflammation was 42.5-44.4\% [3]. The frequency of re-injuries in different studies varied from 10.5 to $27.4 \%$, while using $\operatorname{MSCs}[6,7,29,30]$. It has been shown that PRP is able to release the growth factors that strengthen the tendon lesions healing by stimulating angiogenesis and enhancing the proliferation of MSCs $[26,27]$. By the MSCs and PRP combinations, many researchers have obtained positive clinical results, and the frequency of re-injuries has decreased [11, 28-30]. So in 2013, Ricco et al. showed that as a result of the application of allogeneic AT-MSCs in combination with PRP, 89.5 $\%$ of horses suffering from acute or subacute SDFT tension were able to return to their previous sporting level [29]. Despite the promising results of using autologous MSCs, their rate of application is limited by the spent of cultivated time and as a practical alternative, the allogeneic MSCs have been suggested to use. The use of allogeneic cells makes it possible beforehand to prepare and characterize MSCs and if it is necessary to apply without loss of time. The studies have shown that side effects from the use of allogeneic cells in animals have not been identified and the results are comparable with autologous MSCs $[11,15,16,28,30]$. The cell therapy should not only reduce the regeneration period and improve the quality of the healing process, its main goal is not simple scarring but in the regeneration of fully functional tissue [26]. The presented studies show that MSCs are able to regenerate the tendon. However, 
it is still inferior to the native tissue which indicates the need for further optimization of the protocols for their use. Application of MSCs for equine tendon regeneration is a perspective area. However, it is complicated to objectively evaluate the efficacy of this therapy, since there are differences at different stages of the studies as from the source of MSCs, the model of injury (natural / induced) and its size, to the presence of control groups, the duration of the study, the number of injected cells and possible combinations with other cells. MSCs can be extremely effective in treating equine tendon injuries, however, their actual therapeutic potential is still unclear and there are gaps in knowledge that need to be studied.

\section{Conflict of Interest}

The authors have no conflicts of interest.

\section{References}

[1] Bavin, E. P., et al. (2015). Equine Induced Pluripotent Stem Cells Have a Reduced Tendon Differentiation Capacity Compared to Embryonic Stem Cells. Frontiers in Veterinary Science, vol. 2, p. 55.

[2] Shojaee, A. and Parham, A. (2019). Strategies of Tenogenic Differentiation of Equine Stem Cells for Tendon Repair: Current Status and Challenges. Stem Cell Research and Therapy, vol. 10, issue 1, p. 181.

[3] Dyson, S. J. (2004). Medical Management of Superficial Digital Flexor Tendonitis: A Comparative Study in 219 Horses (1992-2000). Equine Veterinary Journal, vol. 36, issue 5, pp. 415-419.

[4] Savchenkova, I. P., et al. (2010). Methodical Instructions on the Selection of Multipotent Mesenchymal Stem Cells from the Tissues of Adult Mammals, the study of their properties and signs. Publishing House Sputnik+, p. 22.

[5] Smith, R. K., et al. (2003). Isolation and Implantation of Autologous Equine Mesenchymal Stem Cells from Bone Marrow into the Superficial Digital Flexor Tendon as a Potential Novel Treatment. Equine Veterinary Journal, vol. 35, issue 1, pp. 99-102.

[6] Smith, R. K. (2008). Mesenchymal Stem Cell Therapy for Equine Tendinopathy. Disability and Rehabilitation, vol. 30, issue 20-22, pp. 1752-1758.

[7] Godwin, E. E., et al. (2012). Implantation of Bone Marrow-Derived Mesenchymal Stem Cells Demonstrates Improved Outcome in Horses with Overstrain Injury of the 
Superficial Digital Flexor Tendon. Equine Veterinary Journal, vol. 44, issue 1, pp. 25-32.

[8] Nixon, A. J., et al. (2008). Effect of Adipose-Derived Nucleated Cell Fractions on Tendon Repair in Horses with Collagenase-Induced Tendinitis. American Journal of Veterinary Research, vol. 69, issue 7, pp. 928-937.

[9] Carvalho, A. M., et al. (2011). Use of Adipose Tissue-Derived Mesenchymal Stem Cells for Experimental Tendinitis Therapy in Equines. Journal of Equine Veterinary Science, vol. 31, issue 1, pp. 26-34.

[10] Vasileva, S. A. (2019). Characterization of Multipotent Mesenchymal Stem Cells Isolated from Equine Adipose Tissue and Umbilical Cord Blood. Actual Biotechnology, vol. 3, issue 30, pp. 298-300.

[11] Beerts, C., et al. (2017). Tenogenically Induced Allogeneic Peripheral Blood Mesenchymal Stem Cells in Allogeneic Platelet-Rich Plasma: 2-Year Follow-Up after Tendon or Ligament Treatment in Horses. Frontiers in Veterinary Science, vol. 26, issue 4, p. 158.

[12] Longhini, A. L. F., et al. (2019). Peripheral Blood-Derived Mesenchymal Stem Cells Demonstrate Immunomodulatory Potential for Therapeutic Use in Horses. PLoS One, vol. 14, issue 3, p. e0212642.

[13] Prado, A. A., et al. (2015). Characterization of Mesenchymal Stem Cells Derived from the Equine Synovial Fluid and Membrane. BMC Veterinary Research, vol. 11, p. 281.

[14] Koch, T. G., et al. (2007). Isolation of Mesenchymal Stem Cells from Equine Umbilical Cord Blood. BMC Biotechnology, vol. 7, p. 26.

[15] Kang, J. G., et al. (2013). Characterization and Clinical Application of Mesenchymal Stem Cells from Equine Umbilical Cord Blood. Journal of Veterinary Science, vol. 14, issue 3 , pp. 367-371. 